\title{
STUDIES ON SOFT HEMIRINGS AND ITS APPLICATION IN GRAPH THEORY
}

\author{
Md. Yasin Ali1 ${ }^{1 *}$, Kanak Ray Chowdhury ${ }^{3}$, Abeda Sultana ${ }^{2}$, Nirmal Kanti Mitra ${ }^{4}$ \\ ${ }^{1}$ School of Science and Engineering, University of Information Technology \& Sciences, Dhaka, Bangladesh \\ 'Department of Mathematics, Jahangirnagar University, Savar, Bangladesh \\ ${ }^{3}$ Department of Mathematics, Mohammadpur Model School and College, Mohammadpur, Dhaka, Bangladesh \\ ${ }^{4}$ Department of Mathematics and Statistics, Bangladesh University of Business and Technology, Dhaka, Bangladesh \\ *Corresponding Author Email: ali.mdyasin56@gmail.com
}

This is an open access article distributed under the Creative Commons Attribution License, which permits unrestricted use, distribution, and reproduction in any medium, provided the original work is properly cited.

\section{ARTICLE DETAILS}

\section{Article History:}

Received 26 June 2018

Accepted 2 July 2018

Available online 1 August 2018

\section{ABSTRACT}

Molodtsov introduced the concept of soft set, as a mathematical tool for dealing with uncertainties. In this work, we have extended the concept of soft set in hemirings. We have studied soft hemirings and investigated some structural properties of them. Finally using these structural properties, we have characterized graph with loops.

\section{KEYWORDS}

Hemirings, Soft set, Soft hemiring, Graph, Graph with loops.

\section{INTRODUCTION}

After the introduction of soft set in 1999, a host of scholars applied this notion in field of economics, engineering, medical science, social science and many other fields where uncertainties exist [1]. Recently the theoretical studies of soft set are making progress rapidly. In other study, they defined some fundamental operations of soft set such as equality of two soft sets, subset and super set of a soft set, complement of a soft set, null soft set and absolute soft set with examples [2]. The theoretical aspects of soft set were also studied by some researchers [3-10]. In algebra; the concept of soft set was first introduced by researches [11]. They defined soft group and investigated some properties of its. Since then this concept was expanded in many branches of algebraic structures [1122]. On the other hand, the concept of hemiring was first introduced by D. R. LaTorre in 1965; as a special kind of semiring [23]. In 1999, J.S. Golan redefined hemiring and discussed some special classes of hemirings [24]. Later many researchers studied hemirings such as [25-28]. In this paper, first we have studied soft hemirings which is a combination of soft sets and hemirings and investigated some of its fundamental properties [29]. Later we have represented a graph with loop by neighborhood of vertices and soft hemiring and described some properties of graph with loops by using the structural properties of soft hemirings.

\section{PRELIMINARIES}

In this section, we will recall some useful definitions which will be helpful in further pursuit of this study.

Definition 2.1: [27] A hemiring is a nonempty set $H$ on which operations of addition and multiplication have been defined such that the following conditions are satisfied:

- $\quad(H ;+)$ is a commutative monoid with identity element 0 ;

- $(H ; \cdot)$ is a semigroup;

- Multiplication distributes over addition from either side;

- The element 0 is the absorbing element of the multiplication i.e., 0 . $r=0=r \cdot 0$.

A non-empty subset $A$ of $H$ is called a subhemiring of $H$ if it contains zero and is closed with respect to the addition and multiplication of $H$.

Definition 2.2: [30] A graph $G=(V, E)$ consists of a non-empty set of objects $V$, called vertices and a set $E$ of two elements subset of $V$ called edges.
Definition 2.3: [30] If $G=(V, E)$ is a graph and $v \in V$ and $e=\{v\}$, then edge $e$ is called a self-loop. That is, any edge that is a single element subset of $V$ is called a self-loop.

\section{SOME STRUCTURES OF SOFT HEMIRINGS}

Let $H$ be a hemiring and $A$ be two nonempty set. We think about arbitrary binary relation $R$ between an element of $A$ and an element of $H$, that is, $R$ is a subset of $A \times H$ without otherwise defined. A set-valued function $F: A \rightarrow P(H)$ which is defined as

$$
F(a)=\{x \in H:(a, x) \in R\} \text { for all } a \in A .
$$

Then the pair $(F, A)$ is called a soft set of $H$, which is consequent as of the relation $R$. For a soft set $(F, A)$, the set $\operatorname{Supp}(F, A)=\{a \in A: F(a) \neq \emptyset\}$ is called the support of the soft set. A soft set is called null soft set if the support is empty, and we state that a soft set is non-null if $\operatorname{Supp}(F, A) \neq$ $\emptyset$.

Definition 3.1: Let $(H ;+$,$) be a hemiring and A$ be a non-empty set. Then a non-null soft set $(F, A)$ is called a soft hemiring of $H$, if $F(a)$ is a subhemiring of $H$ for all $a \in \operatorname{Supp}(F, A)$.

Example 3.1(a): Let $\left(H=\{0,1,2,3,4,5\} ;+_{6}, \times_{6}\right)$ be a hemiring and $A=\{0,1,2,3,4,5\}$. We consider a set valued function $F: A \rightarrow P(H)$ which is defined as

$$
F(a)=\left\{x \in H: a R x \Leftrightarrow a \times_{6} x \in\{0,2,4\}\right\} \text { for all } a \in A .
$$

Then $F(0)=H, F(1)=\{0,2,4\}, F(2)=H, F(3)=\{0,2,4\}, F(4)=H$ and $F(5)=\{0,2,4\}$.

As we observe that all of these sets are subhemirings of $H$. So $(F, A)$ is a soft hemiring of $H$.

Definition 3.2: Let $(F, A)$ and $(G, B)$ be two soft hemirings of a hemiring $(H ;+$,$) . Then the soft hemiring (G, B)$ is called a soft subhemiring of $(F, A)$ if it satisfies:

$$
\begin{array}{ll}
\text { (i) } & B \subseteq A \text {; } \\
\text { (ii) } & G(a) \text { is a subhemiring of } F(a) \text { for all } a \in \operatorname{Supp}(G, B) .
\end{array}
$$

Example 3.2(a): Let $H=\{0,1,2,3\}$ be a hemiring with the operations table in the table 1. 
Table1: The operations table of the hemiring $H$

\begin{tabular}{c|lllll|llll}
+ & 0 & 1 & 2 & 3 & & 0 & 1 & 2 & 3 \\
\hline 0 & 0 & 1 & 2 & 3 & 0 & 0 & 0 & 0 & 0 \\
1 & 1 & 1 & 2 & 3 & 1 & 0 & 1 & 1 & 1 \\
2 & 2 & 2 & 2 & 3 & 2 & 0 & 1 & 2 & 2 \\
3 & 3 & 3 & 3 & 3 & 3 & 0 & 1 & 2 & 3
\end{tabular}

Let $A=H$ and $F: A \rightarrow P(H)$ is a set valued function defined by

$F(a)=\{x \in H: a R x \Leftrightarrow a \cdot x \leq x\}$ for all $a \in A$.

Then $F(0)=H, F(1)=H, F(2)=H$ and $f(3)=H$. Hence $(F, A)$ is a soft hemiring of $H$.

Let $B=\{0,1,2\}$ be a subset of $A$ and let $G: B \rightarrow P(H)$ be set valued function defined by

$$
G(b)=\{0\} \cup\{y \in H: b R y \Leftrightarrow b+y=b\} \text { for all } b \in B .
$$

Then $G(0)=\{0\}, G(1)=\{0,1\}, G(2)=\{0,1,2\}$ are subhemirings of $F(0), F(1)$ and $F(2)$ respectively. Hence $(G, B)$ is a soft subhemiring of $(F, A)$.

Definition 3.3: Let $(F, A)$ and $(G, B)$ be two soft hemirings of a hemiring $(H ;+, \cdot)$. Then the intersection of $(F, A)$ and $(G, B)$ is represented by $(F, A) \cap(G, B)$ and is defined by

$(F, A) \cap(G, B)=(J, C)$, where $C=A \cap B$

and $J: C \rightarrow P(H)$ is a mapping given by

$J(c)=F(c) \cap G(c)$ for all $c \in C$.

Example 3.3(a): Let $(H=\{0,1,2,3,4,5,6\} ;+, \cdot)$ be a hemiring where the operations are defined in table 2.

Table 2: The operations table of the hemiring $H$

\begin{tabular}{c|lllllllll|lllllll}
+ & 0 & 1 & 2 & 3 & 4 & 5 & 6 & & $\cdot$ & 0 & 1 & 2 & 3 & 4 & 5 & 6 \\
\hline 0 & 0 & 1 & 2 & 3 & 4 & 5 & 6 & & 0 & 0 & 0 & 0 & 0 & 0 & 0 & 0 \\
1 & 1 & 2 & 3 & 4 & 5 & 6 & 4 & & 1 & 0 & 1 & 2 & 3 & 4 & 5 & 6 \\
2 & 2 & 3 & 4 & 5 & 6 & 4 & 5 & 2 & 0 & 2 & 4 & 6 & 5 & 4 & 6 \\
3 & 3 & 4 & 5 & 6 & 4 & 5 & 6 & & 3 & 0 & 3 & 6 & 6 & 6 & 6 & 6 \\
4 & 4 & 5 & 6 & 4 & 4 & 5 & 6 & & 4 & 0 & 4 & 5 & 6 & 4 & 5 & 6 \\
5 & 5 & 6 & 4 & 5 & 6 & 4 & 5 & 5 & 0 & 5 & 4 & 6 & 5 & 4 & 6 \\
6 & 6 & 4 & 5 & 6 & 4 & 5 & 6 & 6 & 0 & 6 & 6 & 6 & 6 & 6 & 6
\end{tabular}

Let $A=\{0,1\}$ and $F: A \rightarrow P(H)$ is a set valued function defined by

$F(a)=\{x \in H: a R x \Leftrightarrow a \cdot x \in(0,3,6)\}$ for all $a \in A$.

Then $F(0)=H$ and $F(1)=\{0,3,6\}$, which are subhemirings of $H$, and therefore $(F, A)$ is a soft hemiring of $H$.

Again let $B=\{1,2\}$ and $G: B \rightarrow P(H)$ be a set valued function defined by

$G(b)=\{x \in H: a R x \Leftrightarrow b \cdot x=(0,6)\}$ for all $b \in B$.

Then $G(1)=\{0,6\}$ and $G(2)=\{0,3,6\}$, which are subhemirings of $H$ and so $(G, B)$ is also a soft hemiring of $H$.

Now, $(F, A) \cap(G, B)=(J, C)$ where $C=A \cap B=\{1\}$, and for all $c \in C$, we have $J(1)=\{0,6\}$.

Theorem 3.4: Let $(F, A)$ and $(G, B)$ be two soft hemirings of a hemiring $(H ;+, \cdot)$. Then $(F, A) \cap(G, B)$ is also a soft hemiring of $H$ if it is non-null.

Proof: By definition 3.3, we can write

$$
(F, A) \cap(G, A)=(J, C)
$$

where $C=A \cap B \neq \varnothing$

and $J(c)=F(a) \cap G(b)$ for all $c \in C$.

By hypothesis $(J, C)$ is a non-null soft set of $H$, so $\forall x \in \operatorname{Supp}(J, C)$, we have $J(c)=F(a) \cap G(b) \neq \emptyset$.

Thus the non-empty sets $F(a)$ and $G(b)$ are both subhemirings of $H$. Hence
$J(c)$ is a subhemiring of $H$ for all $c \in \operatorname{Supp}(J, C)$ and so $(J, C)=(F, A) \cap$ $(G, B)$ is a soft hemiring of $H$ as required.

Corollary 3.5: Let $(F, A)$ and $(G, A)$ be two soft hemirings of a hemiring $(H ;+$,$) . Then we have the following:$

The bi-intersection $(F, A) \cap(G, A)$ is a soft hemiring of $H$ if it is non-null.

$(F, A) \cap(G, A)$ is a soft subhemiring of $(F, A)$ and $(G, A)$ if it is non-null.

\section{Proof: Trivial}

Definition 3.6: Let $(F, A)$ and $(G, B)$ be two soft hemirings of a hemiring $(H ;+, \cdot)$, then the union of $(F, A)$ and $(G, B)$ is represented by $(F, A) \cup(G, B)$ and is defined by $(F, A) \cup(G, B)=(J, C)$, where $C=$ $A \cup B$ and $J: C \rightarrow P(H)$ is a mapping given by

$$
J(c)=\left\{\begin{array}{c}
F(c) \text { if } c \in A-B \\
G(c) \text { if } c \in B-A \\
F(c) \cup G(c) \text { if } c \in A \cap B
\end{array}\right.
$$

for all $c \in C$.

Theorem 3.7: Let $(F, A)$ and $(G, B)$ be two soft hemirings of a hemiring $(H ;+$,$) . If A$ and $B$ are disjoint, then $(F, A) \cup(G, B)$ is a soft hemiring of $H$.

Proof: We have from the definition of 3.6.

$$
J(c)=\left\{\begin{array}{c}
F(c) \text { if } c \in A-B ; \\
G(c) \text { if } c \in B-A ; \\
F(c) \cup G(c) \text { if } c \in A \cap B .
\end{array}\right.
$$

for all $c \in C$. Since $A \cap B=\emptyset$, it follows that $c \in A-B$ or $c \in B-A$ for all $c \in C$. If $c \in A-B$, then $J(c)=F(c)$ is a subhemiring of $H$ since $(F, A)$ is a soft hemiring of $H$. If $c \in B-A$, then $J(c)=G(c)$ is a subhemiring of $H$ since $(G, B)$ is a soft hemiring of $H$. Hence $(F, A) \cup(G, A)$ is a soft hemiring of $H$.

If $A \cap B \neq \varnothing$, in the theorem 3.7, then the theorem 3.7 is not true in general as shown in the following example.

Example 3.8: Let $\left(H=\{0,1,2,3,4,5\} ;{ }_{6}, \times_{6}\right)$ be a hemiring.

Let $A=\{3,4\}$ and $F: A \rightarrow P(H)$ be a set valued function defined by

$F(a)=\{x \in H: a R x \Leftrightarrow a \cdot x=0\}$ for all $a \in A$.

Then $F(3)=\{0,2,4\}$ and $F(4)=\{0,3\}$, and therefore $(F, A)$ is a soft hemiring of $H$.

Again let $B=\{4\}$ and $G: B \rightarrow P(H)$ be a set valued function defined by

$$
G(b)=\{x \in H: a R x \Leftrightarrow b+x=\{0,2\}\}
$$

for all $b \in B$.

Then $G(4)=\{0,2,4\}$, and therefore $(G, B)$ is also a soft hemiring of $H$.

Now $J(4)=F(4) \cup G(4)=\{0,2,3,4\}$, which is not subhemiring of $H$, since $2+3=5 \notin J(4)$, but $2,3 \in J(4)$.

Definition 3.9: Let $(F, A)$ and $(G, B)$ be two soft hemirings of a hemiring $(H ;+, \cdot)$, then " $(\boldsymbol{F}, \boldsymbol{A}) \boldsymbol{A} \boldsymbol{N D}(\boldsymbol{G}, \boldsymbol{B})$ " represented by $(F, A) \wedge(G, B)$ and is defined by $(F, A) \wedge(G, B)=(J, C)$ where $C=A \times B$ and $J(a, b)=$ $F(a) \cap G(b)$ for all $(a, b) \in C$.

Example 3.9(a): Consider the soft hemirings $(F, A)$ and $(G, B)$ in the example 3.3(a).

Now $(F, A) \wedge(G, B)=(J, C)$,

where $C=A \times B=\{(0,1),(0,2),(1,1),(1,2)\}$ and for all $(a, b) \in C=A \times$ $B$, we have

$$
\begin{aligned}
& J(0,1)=F(0) \cap G(1)=\{0,6\}, \\
& J(0,2)=F(0) \cap G(2)=\{0,3,6\}, \\
& J(1,1)=F(1) \cap G(1)=\{0,6\}, \\
& J(1,2)=F(1) \cap G(2)=\{0,3,6\} .
\end{aligned}
$$

Theorem 3.10: Let $(F, A)$ and $(G, B)$ be two soft hemirings of a hemiring $(H ;+, \cdot)$, then $(F, A) \wedge(G, B)$ is a soft hemiring of $H$, if it is nonnull. 
Proof: Let $(F, A) \wedge(G, A)=(J, C)$, where $J(a, b)=F(a) \cap G(b)$ for $\operatorname{all}(a, b) \in C$. Then by hypothesis, $(J, C)$ is a non-null soft set of $H$, so for all $(a, b) \in \operatorname{Supp}(J, C)$, we have

$J(c)=F(c) \cap G(c) \neq \varnothing$.

It follows that $F(c) \neq \varnothing$ and $G(c) \neq \varnothing$ are both subhemirings of $H$.

Hence $J(a, b)$ is a subhemiring of $H$ for all $(a, b) \in \operatorname{Supp}(J, C)$ and so $(J, C)=(F, A) \wedge(G, B)$ is a soft hemiring of $H$ as required.

Definition 3.11: Let $(F, A)$ and $(G, B)$ be two soft hemirings of a hemiring $(H ;+, \cdot)$, then " $(\boldsymbol{F}, \boldsymbol{A}) \boldsymbol{O} \boldsymbol{R}(\boldsymbol{G}, \boldsymbol{B})$ " represented by $(F, A) \vee(G, B)$ is defined by $(F, A) \vee(G, B)=(J, C)$ where $C=A \times B$ and $J(a, b)=$ $F(a) \cup G(b)$ for all $(a, b) \in C$.

Example 3.11(a): Consider the soft hemirings $(F, A)$ and $(G, B)$ in the example 3.3(a)

Now, $(F, A) \vee(G, B)=(J, C)$,

where $C=A \times B=\{(0,1),(0,2),(1,1),(1,2)\}$

and for all $(a, b) \in C$, we have

$$
\begin{aligned}
& J(0,1)=F(0) \cup G(1)=H, \\
& J(0,2)=F(0) \cup G(2)=H, \\
& J(1,1)=F(1) \cup G(1)=\{0,3,6\}, \\
& J(1,2)=F(1) \cup G(2)=\{0,3,6\} .
\end{aligned}
$$

Theorem 3.12: Let $(F, A)$ and $(G, B)$ be two soft hemirings of a hemiring $(H ;+, \cdot)$, then $(F, A) \vee(G, B)$ is a soft hemiring of $H$, if it is nonnull.

\section{Proof: Trivial}

\section{CHARACTERIZATION OF GRAPH BY SOFT HEMIRING}

In this section, we have introduced a method to represent a graph with loops which is based on neighborhood of vertices and soft hemiring of a hemiring.

Proposition 4.1: Every graph can be represented by a soft hemiring of a hemiring.

Proof: Let $G=(H, E)$ be a graph with $n$ vertices and $m$ edges, where $H$ be a hemiring whose elements represent $n$ vertices and $E$ be the set of $m$ edges. Now define a map $F: H \rightarrow P(H)$ such that for all $a \in H$,

$F(a)=\{x \in H$, such that $x$ is adjacent to $a\}$

That is the subset $F(a)$ of $H$ containing all vertices adjacent to $a$. If $F(a)$ is a subhemiring of $H$, then a soft set $(F, H)$ is a soft hemiring of $H$ which represents the graph $G=(H, E)$

Lemma 4.2: A graph $G$ represented by a soft hemiring $(F, H)$ of a hemiring $H$ has a loop at $a \in H$ if and only if $a \in F(a) ; \forall a \in \operatorname{Supp}(F, H)$.

Theorem 4.3: Let $H$ be a hemiring. Then every soft hemiring $(F, H)$ of a hemiring $H$ represents a graph with loop.

Proof: Let $G=(H, E)$ be a graph with $n$ vertices and $m$ edges, and $H$ be a hemiring whose elements represent $n$ vertices and $E$ be the set of $m$ edges. Now define a map $F: H \rightarrow P(H)$ such that for all $a \in H$,

$F(a)=\{x \in H$, such that $\mathrm{x}$ is adjacent to $a\}$

That is the subset $F(a)$ of $H$ containing all vertices adjacent to $a$. If $F(a)$ is a subhemiring of $H$, then a soft set $(F, H)$ is a soft hemiring of $H$ which represents the graph $G=(H, E)$. On the other hand, since $F(a)$ is a subhemiring of $H$, so $0 \in H \Rightarrow 0 \in F(0)$. In this case there arises a loop. This concludes the proof

Example 4.3(a): Let $\left(H=\{0,1,2,3,4,5\} ;+_{6}, \times_{6}\right)$ be a hemiring. We consider a set valued function $F: H \rightarrow P(H)$ which is defined as,

$$
F(a)=\left\{x \in H: a R x \Leftrightarrow a \times_{6} x=0\right\} \text { for all } a \in H .
$$

Then,

$F(0)=H, F(1)=\{0\}, F(2)=\{0,3\}, F(3)=\{0,2,4\}, F(4)=$

$\{0,3\}$ and $F(5)=\{0\}$. As we observe that all of these sets are subhemirings of $H$. So $(F, A)$ is a soft hemiring of $H$. And by the rule of neighborhood we have the graph $G$ with loop is shown in figure 1.

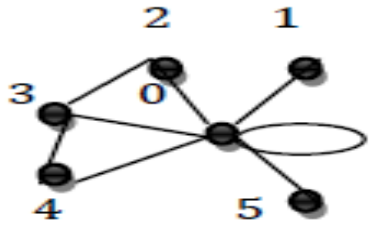

Figure 1: Graph with loop $G$

Note: From now, we will use graph instead of graph with loop.

Definition 4.4: Let $G$ be a graph represented by a soft hemiring $(F, H)$ of a hemiring $H$. Then the degree of any vertex $a$ is the number of vertices adjacent to $a$. For a loop of any vertex the degree is 2

Theorem 4.5: Let $G$ be a graph represented by a soft hemiring $(F, H)$ of a hemiring $H$. Then the degree of $G$ is $\sum_{a_{i} \in H}\left|F\left(a_{i}\right)\right|+l$, where $\left|F\left(a_{i}\right)\right|$ denote the cardinality of $F\left(a_{i}\right)$ and $l$ denote the number of loop.

Proof: Let $a_{i} \in H$ be a vertices of the graph $G$. If $a_{i}$ has no loop, then $a_{i}$ is adjacent to each element of $F\left(a_{i}\right)$. Then the degree is $\sum_{a_{i} \in H}\left|F\left(a_{i}\right)\right|$. If for all $a_{i} \in H$ there is any $a_{i} \in F\left(a_{i}\right)$, then there will be a loop and the degree is $\sum_{a_{i} \in H}\left|F\left(a_{i}\right)\right|+1$. If there are $l$ number loops in the graph then the degree is $\sum_{a_{i} \in H}\left|F\left(a_{i}\right)\right|+l$.

Example 4.5(a): In the example 4.3(a). We have $|F(0)|=6,|F(1)|=$ $1,|F(2)|=2,|F(3)|=3,|F(4)|=2,|F(5)|=1$. Also there is a loop. So the degree of the graph $G$ is $6+1+2+3+2+1+1=16$

Corollary 4.6: Let $G$ be a graph represented by a soft hemiring $(F, H)$ of a hemiring $H$. Then $G$ has even number vertices of an even degree.

Proof: Trivial

Example 4.7: Consider the example 4.3(a). There are 6 vertices when the degree is 16.

Definition 4.8: Let $G_{1}$ and $G_{2}$ be two graphs represented by soft hemirings $(F, H)$ and $(K, H)$ respectively of a hemiring $H$. Then $G_{2}$ is a subgraph of $G_{1}$ if $(K, H) \subseteq(F, H)$.

Example 4.8(a): The set $H=\{0, a, b\}$ is a hemiring with the operations table in table 3 .

Table 3: The operations table of the hemiring $H$

\begin{tabular}{c|ccccc|ccc}
+ & 0 & $a$ & $b$ & & $\cdot$ & 0 & $a$ & $b$ \\
\hline 0 & 0 & $a$ & $b$ & & 0 & 0 & 0 & 0 \\
$a$ & $a$ & $a$ & $b$ & & $a$ & 0 & $a$ & $a$ \\
$b$ & $b$ & $b$ & $b$ & & $b$ & 0 & $a$ & $b$
\end{tabular}

Let $F: H \rightarrow P(H)$ is a set valued function defined by

$$
F(e)=\{x \in H: e R x \Leftrightarrow d(e, x) \leq 1\} \text { for all } e \in H .
$$

That is, $F(0)=H, F(a)=H$ and $F(b)=H$. We notice that all of these sets are subhemirings of $H$. Hence $(F, H)$ be a soft hemiring of $H$. And by the rule neighborhood the graph is as follows:

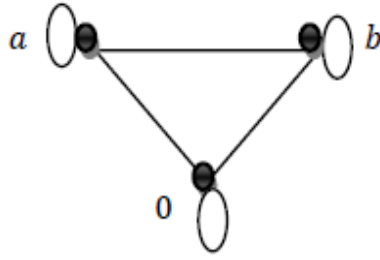

Figure 2: Graph $G_{1}$

Again let $K: H \rightarrow P(H)$ is a set valued function defined by

$$
K(e)=\{0\} \cup\{y \in H: e R y \Leftrightarrow d(e, y)=1\} \text { for all } e \in H .
$$

That is,

$K(0)=H, K(a)=\{0, b\}$ and $K(b)=\{0, a\}$.

We examine that all of these sets are subhemirings of $H$. Hence $(K, H)$ be a soft hemiring of $H$. And the graph corresponding to this soft hemiring $(K, H)$ is as follows: 


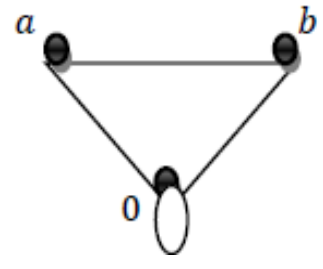

Figure 3: Graph $G_{2}$

We stain that $(K, H) \subseteq(F, H)$.

Hence $G_{2}$ is a subgraph of $G_{1}$.

Definition 4.9: Let $G_{1}$ and $G_{2}$ be two graphs represented by the soft hemirings $(F, H)$ and $(K, H)$ respectively. Then the bi-intersection of $G_{1}$ and $G_{2}$ is indicated by $G=G_{1} \cap G_{2}$ is a soft hemiring $(J, H)$ where $J(a)=$ $F(a) \cap K(a)$ for all $a \in H$.

Example 4.9(a): Consider the graph $G_{1}$ and $G_{2}$ represented by the soft hemirings $(F, H)$ and $(K, H)$ respectively of a hemiring $H$ in the example $4.8(\mathrm{a})$.

Here,

$$
\begin{aligned}
& J(0)=F(0) \cap K(0)=H, \\
& J(a)=F(a) \cap K(a)=\{0, b\}, \\
& J(b)=F(b) \cap K(b)=\{0, a\} .
\end{aligned}
$$

Therefore $(J, H)$ is also a soft hemiring and the graph $G=G_{1} \cap G_{2}$ is given in the figure 4.

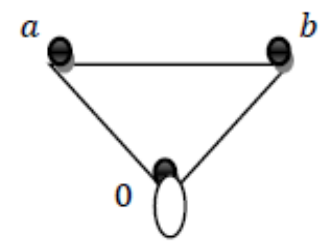

Figure 4: Graph of $G_{1} \cap G_{2}$

Definition 4.10: Let $G_{1}$ and $G_{2}$ be two graphs represented by the soft hemirings $(F, H)$ and $(K, H)$ respectively. Then the bi-union of $G_{1}$ and $G_{2}$ is denoted by $G=G_{1} \cup G_{2}$ is a soft hemiring $(J, H)$ where $J(a)=F(a) \cup$ $K(a)$ for all $a \in H$.

Example 4.10(a): Consider the graph $G_{1}$ and $G_{2}$ represented by the soft hemirings $(F, H)$ and $(K, H)$ respectively of a hemiring $H$ in the example 4.8(a). Here $J(0)=F(0) \cup K(0)=H, J(a)=F(a) \cup K(a)=H, J(b)=F(b)$ $\cup K(b)=H$. Therefore $(J, H)$ is also a soft hemiring and the graph $G=G_{1}$ $\cup G_{2}$ is given in the figure 5 .

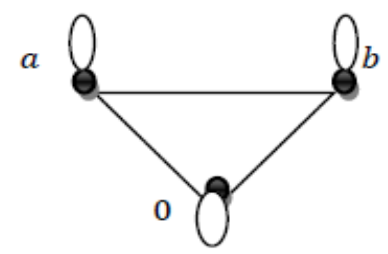

Figure 5: Graph $G=G_{1} \cup G_{2}$

\section{CONCLUSION}

After the introduction of soft set, it became very effective mathematical tools in our daily life problems where uncertainties exist. With the rapid applications of soft set in many branches of science and engineering, a host of scholars also broad this technique in numerous branches of mathematics. In this work, we have extended the concept of soft sets to the structures of hemirings as soft hemirings and studied some of its basic properties. Finally, we have applied these structures in graph theory; basically a graph which has loops. This works will be helpful to the reader as well as researcher for further study of soft hemiring and its application in many branches of mathematics and other engineering branches.

\section{REFERENCES}

[1] Molodtsov, D. 1999. Soft set theory - First results. Computers and Mathematics with Applications, 37, 19-31.
[2] Maji, P.K., Biswas, R., Roy, A.R. 2003. Soft set theory. Computers and Mathematics with Applications, 45, 555-562.

[3] Ali, M.I., Feng, F., Liu, X., Min, W.K. 2009. On some new operations in soft set theory. Computers and Mathematics with Applications, 57 (9), 1547-1553.

[4] Babitha, K.V., Sunil, J.J. 2010. Soft set relations and functions. Computers and Mathematics with Applications, 60 (7), 1840-1849.

[5] Majumdar, P., Samanta, S.K. 2010. On soft mappings. Computers and Mathematics with Applications, 60 (9), 2666-2672.

[6] Xiao, Z., Gong, K., Xia, S., Zou, Y. 2010. Exclusive disjunctive soft sets. Computers and Mathematics with Applications, 59, 2128-2137.

[7] Qin, K., Hong, Z. 2010. On soft equality. Journal of Computational and Applied Mathematics, 234, 1347-1355.

[8] Gong, K., Xiao, Z., Zhang, X. 2010. The bijective soft set with its operations. Computers and Mathematics with Applications, 60, 22702278.

[9] Sezgin, A., Atagün, A.0. 2011. On operations of soft sets. Computers and Mathematics with Applications, 61, 1457-1467.

[10] Park, J.H., Kima, O.H., Kwunb, Y.C. 2012. Some properties of equivalence soft set relations. Computers and Mathematics with Applications, 63, 1079-1088.

[11] Aktas, H., Cagman, N. 2007. Soft sets and soft groups. Information Sciences, 177, 2726- 2735.

[12] Jun, Y.B. 2008. Soft BCK/BCI-algebras. Computers and Mathematics with Applications, 56, 1408-1413.

[13] Feng, F., Jun, Y.B., Zhao, X. 2008. Soft semirings. Computers and Mathematics with Applications, 56 (10), 2621-2628.

[14] Jun, Y.B., Park, C.H. 2008. Applications of soft sets in ideal theory of BCK/BCI-algebras. Information Sciences, 178, 2466-2475.

[15] Jun, Y.B., Park, C.H. 2009. Applications of soft sets in Hilbert algebras. Iranian Journal of Fuzzy Systems, 6 (2), 75-88.

[16] Jun, Y.B., Lee, K.J., Park, C.H. 2009. Soft set theory applied to ideals in d-algebras. Computers and Mathematics with Applications, 57, 367-378.

[17] Jun, Y.B., Lee, K.J., Zhan, J. 2009. Soft p-ideals of soft BCI-algebras. Computers and Mathematics with Applications, 58, 2060-2068.

[18] Acar, U., Koyuncu, F., Tanay, B. 2010. Soft sets and soft rings. Computers and Mathematics with Applications, 59, 3458-3463.

[19] Kazancı, O., Yılmaz, S., Yamak, S. 2010. Soft sets and soft BCH-algebras. Hacettepe Journal of Mathematics and Statistics, 39 (2), 205-217.

[20] Ozturk, M.A., Inan, E. 2011. Soft $\Gamma$ - rings and idealistic soft $\Gamma$ - rings. Annals of fuzzy Mathematics and informatics, 1 (1), 71-80.

[21] Sezgin, A., Atagün, A.0. 2011. Soft groups and normalistic soft groups. Computers and Mathematics with Applications, 62, 685-698.

[22] Atagün, A.O., Sezgin, A. 2011. Soft substructures of rings, fields and modules. Computers and Mathematics with Applications, 61, 592-601.

[23] La Torre, D.R. 1976. The Brown-McCoy radicals of a hemiring. Publicationes Mathematicae, Debrecen, 14, 15 - 28.

[24] Golan, J.S. 1999. Semirings and their applications, Kluwer Acad. Publ.

[25] Adhikari, M.R., Adhikari, A. 2014. Basic Modern Algebra with Applications, Springer new Delhi Heidelberg New York Dordrect London Library of congress.

[26] Assaye, B., Rao, G.C. 2014. A Characterization of Inverse almost Hemirings. Journal of Mathematical Sciences: Advances and Applications, $27,95-102$.

[27] Ali, M.Y., Chowdhury, K.R., Sultana, A., Mitra, N.K. 2017. Some 
Structures of Hemirings. Pure and Applied Mathematics Journal, 6 (1), 4550 .

[28] Ali, M.Y., Chowdhury, K.R., Sultana, A., Mitra, N.K. 2017. T-Fuzzy Left h-Ideals of Hemirings. Asian Research Journal of Mathematics, 4 (1), 1-14.
[29] Ali, M.I., Shabir, M., Feng, F. 2017. Representation of graphs based on neighborhoods and soft sets. International Journal of Machine Learning and Cybernetics, 8, 1525-1535

[30] Harary, F. 1969. Graph Theory, Addison-Wesley Publishing Company, Inc. 